\title{
A theoretical investigation of the flurbiprofen methyl ester isomerization as the main step in the photopreparation of anti-inflammatory medicine (S)-flurbiprofen: A DFT study
}

\author{
Saba Hadidi ${ }^{a^{*}}$, Mohammadsaleh Norouzibazaz ${ }^{\mathrm{b}, \mathrm{c}}$ and Farshad Shiri ${ }^{\mathrm{a}}$
}

${ }^{a}$ Department of Inorganic Chemistry, Faculty of Chemistry, Razi University, Kermanshah, Iran
${ }^{b}$ Nano Science and Technology Research Center, Razi University, Kermanshah, Iran

${ }^{c}$ Department of Organic Chemistry, Faculty of Chemistry, Razi University, Kermanshah, Iran

\begin{tabular}{l} 
C H R O N I C L E \\
\hline Article history: \\
Received October 8, 2019 \\
Received in revised form \\
November 21, 2019 \\
Accepted February 18,2020 \\
Available online \\
February 18, 2020 \\
\hline Keywords: \\
Flurbiprofen \\
R/S isomerization \\
DFT calculation \\
Conversion mechanism
\end{tabular}

\section{Introduction}

Flurbiprofen, racemic 2-(2-fluoro-4-biphenyl) propionic acid is a famous orally effective nonsteroidal anti-inflammatory drug (NSAID), which is widely used for the treatment of pain due to rheumatoid arthritis, osteoarthritis, ankylosing Spondylitis, and acute gouty arthritis. ${ }^{1,2}$ This medicine has attracted a lot of attention because of its important advantageous properties. The analgesic effects of this medicine are mainly attributed to the inhibition of the enzymatic activity of cyclooxygenase, which leads to the suppression of prostaglandin synthesis. ${ }^{3-5}$ Due to this analgesic effects, flurbiprofen can also be utilized in short term alleviation of post-operative pain in dental patients. ${ }^{5}$ Experiments indicated that flurbiprofen and other NSAIDs can reduce the relative risk of colorectal cancer after two or more years of continuous use. ${ }^{6,7}$ Several investigations revealed that flurbiprofen like other clinically significant medicines shows stereoselectivity in action and disposition. ${ }^{8-11}$ It means that different

* Corresponding author.

E-mail address: saba.hadidi@yahoo.com (S. Hadidi)

(C) 2020 Growing Science Ltd. All rights reserved.

doi: $10.5267 /$ j.ccl.2020.2.002 
enantiomers have various impacts on pharmacokinetic processes. ${ }^{12-14}$ Like other NSAIDs, such as ibuprofen and fenoprofen, the drug with a chiral center, can inhibit cyclooxygenase only by its $(S)$ enantiomer. Another enantiomer of flurbiprofen $((R)$-flurbiprofen) exhibits minimal inhibition cyclooxygenase activity. ${ }^{15}$ Unlike the other NSAIDs, ${ }^{16}$ flurbiprofen does not undergo configuration inversion and the $(R)$-flurbiprofen is not epimerized to the $(S)$-enantiomer in humans. ${ }^{17}$ Enzymes have a significant role in determination the enantiomers of organic synthesis. One of the enzymes that can catalyze the reactions in aqueous solvents in highly enantioselective level is Candida rugosa lipase (EC 3.1.1.3). ${ }^{18,19}$ The enzymes not only are able to hydrolase soluble and insoluble substrates in aqueous conditions, but also can be used widely in organic synthesis. ${ }^{20}$ The current work aims to investigate the isomerization mechanism of racemic flurbiprofen by employing the quantum chemical method. In this study, in order to overcome the theoretical $50 \%$ limit in the resolution of racemic medicines, the facile conversion of racemic flurbiprofen into its $(S)$-enantiomer by dynamic kinetic resolution (DKR) conditions was employed. ${ }^{19}$ The most common transformation-catalyzing lipases were employed to study the kinetic resolution of racemic flurbiprofen. ${ }^{21}$ As illustrated in Figure 1, the computations were carried out by considering esterification of flurbiprofen to relating flurbiprofen methyl ester.

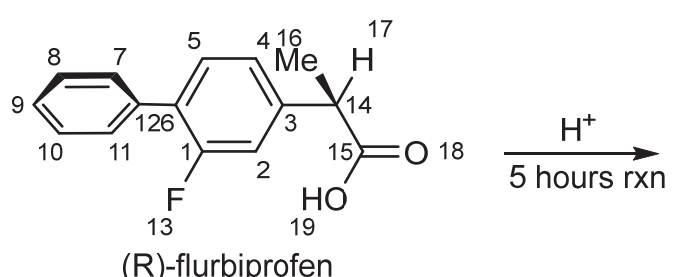

(R)-flurbiprofen

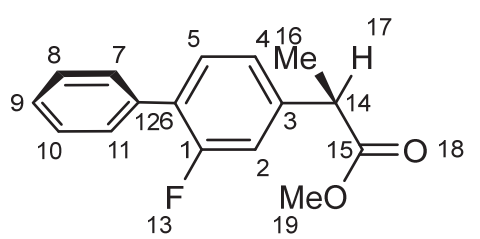

(R)-flurbiprofen methyl ester

$99 \%$ conversion

Fig. 1. Fisher esterification of racemic flurbiprofen in methanol at $40{ }^{\circ} \mathrm{C}$ for $5 \mathrm{~h}$

\section{Results and discussion}

$(R)$-flurbiprofen undergoes an energetically favorable second ground state rearrangement to generate (S)-flurbiprofen (Fig. 2).

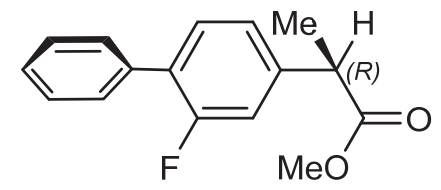

(R)-Flurbiprofen methyl ester

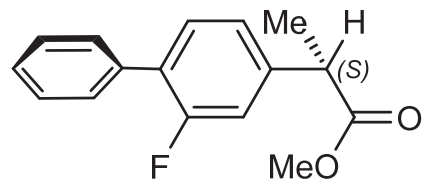

(S)-Flurbiprofen methyl ester

Fig. 2. $R / S$-flurbiprofen enantiomers

The process route leading from $(R)$-flurbiprofen to $(S)$-flurbiprofen corresponding to the inversion of stereochemistry at $\mathrm{C} 14$ have been identified. ${ }^{22}$ The reaction pathway of connecting $(R)$-flurbiprofen methyl ester to $(S)$-flurbiprofen methyl ester is illustrated in Fig. 3 with details in Table 1. The used relative Gibbs free energies are also listed in Table 2. Based upon this pathway, the R isomer undergoes inversion of the stereochemistry or retention of configuration to generate the $(S)$-flurbiprofen or reformation of the primary $(R)$-flurbiprofen methyl ester. The full geometry optimizations along this reaction pathway were carried out at the DFT level by employing the PBE0 level of theory. ${ }^{16}$ The calculations result depicted that the energy of $(R)$-flurbiprofen methyl ester is $1.50 \mathrm{kcal} / \mathrm{mol}$ higher than $(S)$-enantiomer. The first step in the isomerization of flurbiprofen is the excitation of flurbiprofen methyl ester in its initial form to the first excited singlet state S1 since the symmetry of [1,3]-hydrogen shift from $\mathrm{C} 14$ to $\mathrm{O} 18$ is forbidden. ${ }^{23-25}$ 


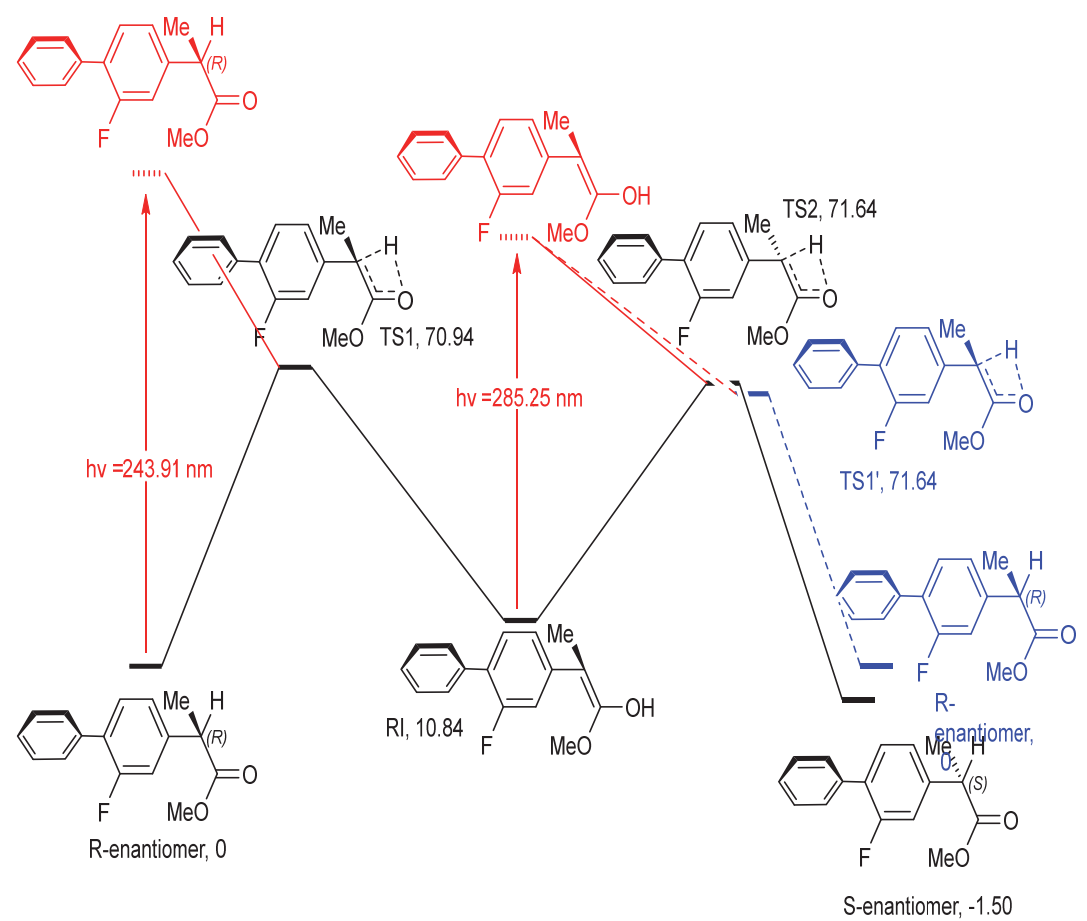

Fig. 3. Relative Gibbs free energy (in $\mathrm{kcal} / \mathrm{mol}$ ) diagram for the photochemical isomerization of flurbiprofen methyl ester in aqueous solution

Table. 1. Bond length (in $\AA$ ) for the photochemical isomerization of flurbiprofen methyl ester in aqueous solution

\begin{tabular}{ccccccccc}
\hline \multicolumn{2}{c}{ Parameter } & \multicolumn{7}{c}{ Critical structure } \\
\hline & $\mathrm{R}$ & $\mathrm{R}^{*}$ & $\mathrm{TS} 1$ & $\mathrm{RI}$ & $\mathrm{RI}^{*}$ & $\mathrm{TS} 1^{\prime}$ & $\mathrm{TS} 2$ & $\mathrm{~S}$ \\
\hline C3-C14 & 1.521 & 1.521 & 1.472 & 1.477 & 1.477 & 1.472 & 1.48 & 1.519 \\
$\mathbf{C 1 4 - C 1 6}$ & 1.529 & 1.529 & 1.531 & 1.513 & 1.513 & 1.531 & 1.516 & 1.527 \\
$\mathbf{C 1 4 - H 1 7}$ & 1.093 & 1.093 & 1.57 & - & - & 1.57 & 1.575 & 1.09 \\
$\mathbf{C 1 4 - C 1 5}$ & 1.517 & 1.517 & 1.445 & 1.35 & 1.35 & 1.445 & 1.448 & 1.517 \\
$\mathbf{C 1 5 - O 1 8}$ & 1.219 & 1.219 & 1.288 & 1.36 & 1.36 & 1.288 & 1.286 & 1.217 \\
$\mathbf{C 1 5 - O 1 9}$ & 1.331 & 1.331 & 1.295 & 1.349 & 1.349 & 1.295 & 1.293 & 1.336 \\
\hline
\end{tabular}

As is evident from Fig. 4, the UV spectrum indicated that the first vertical S1 excitation (HOMO to LUMO) occurs at $\lambda=243.91 \mathrm{~nm}$, with oscillator strength $\mathrm{f}=0.5776$ and one at $\lambda=234.45 \mathrm{~nm}$, with oscillator strength $\mathrm{f}=0.2194$. This finding is in complete agreement with the orbitals demonstrated in Fig. 5 which shows that the excitation is of $\pi \rightarrow \pi^{*}$ transition nature. Furthermore, an additional peak with lower oscillator strength $(\mathrm{f}=0.0140)$ was observed at $\lambda=233.99 \mathrm{~nm}$, assigned to HOMO- 1 to the LUMO.

Table. 2. Relative Gibbs free energy (in $\mathrm{kcal} / \mathrm{mol}$ ) for the photochemical isomerization of flurbiprofen methyl ester in aqueous solution

\begin{tabular}{cccc}
\hline Critical structure & $\boldsymbol{\Delta G}$ & Critical structure & $\boldsymbol{\Delta} \mathbf{G}$ \\
\hline $\mathrm{R}$ & 0 & $\mathrm{RI}^{*}$ & 100.23 \\
$\mathrm{R}^{*}$ & 117.22 & $\mathrm{TS} 1^{\prime}$ & 70.94 \\
$\mathrm{TS} 1$ & 70.94 & $\mathrm{TS} 2$ & 71.64 \\
$\mathrm{RI}$ & 10.83 & $\mathrm{~S}$ & -1.50 \\
\hline
\end{tabular}




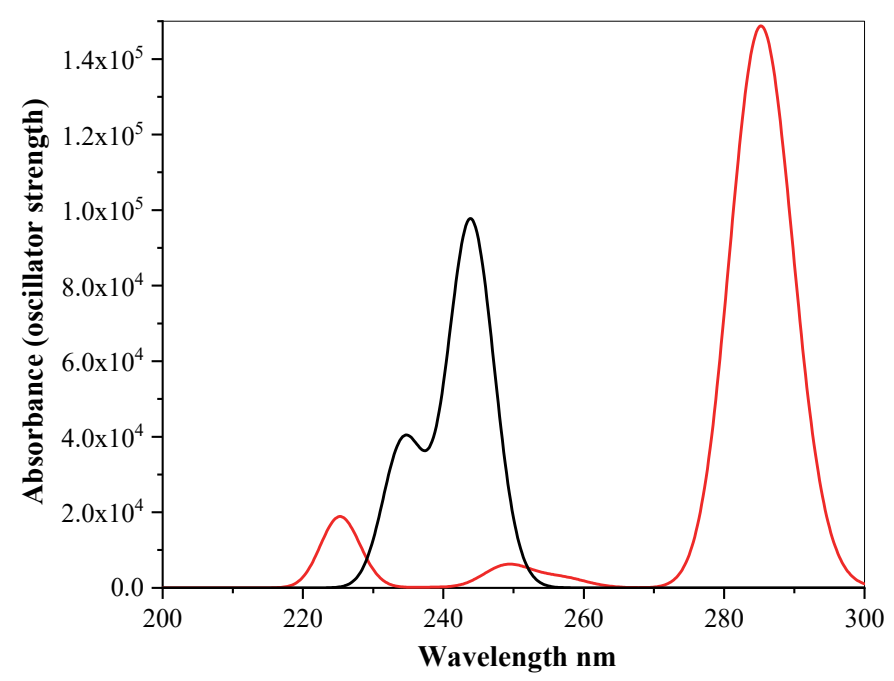

Fig. 4. TD-PBE0/6-31++G(d,p) computed absorption spectra in the range 200-300 $\mathrm{nm}$ of the $(R)-$ flurbiprofen methyl ester (black) and (E)-2-(2-fluoro-[1,1'-biphenyl]-4-yl)-1-methoxyprop-1-en-1-ol (red)

As illustrated in Fig. 3 near the $(R)$-flurbiprofen methyl ester geometry, a transition state, TS1 corresponding to $\mathrm{C} 14-\mathrm{H} 17$ bond breakage has $70.94 \mathrm{kcal} / \mathrm{mol}$ higher energy than the optimized ground state $(R)$-flurbiprofen methyl ester. Since this process involves the migration of $\mathrm{H} 17$ from $\mathrm{C} 14$ to $\mathrm{O} 18$ is considered as a $[1,3]$ Hydrogen shift. ${ }^{26}$ The Intrinsic reaction coordinates (IRC) were calculated at the PBE0 level to authenticate the transition state.

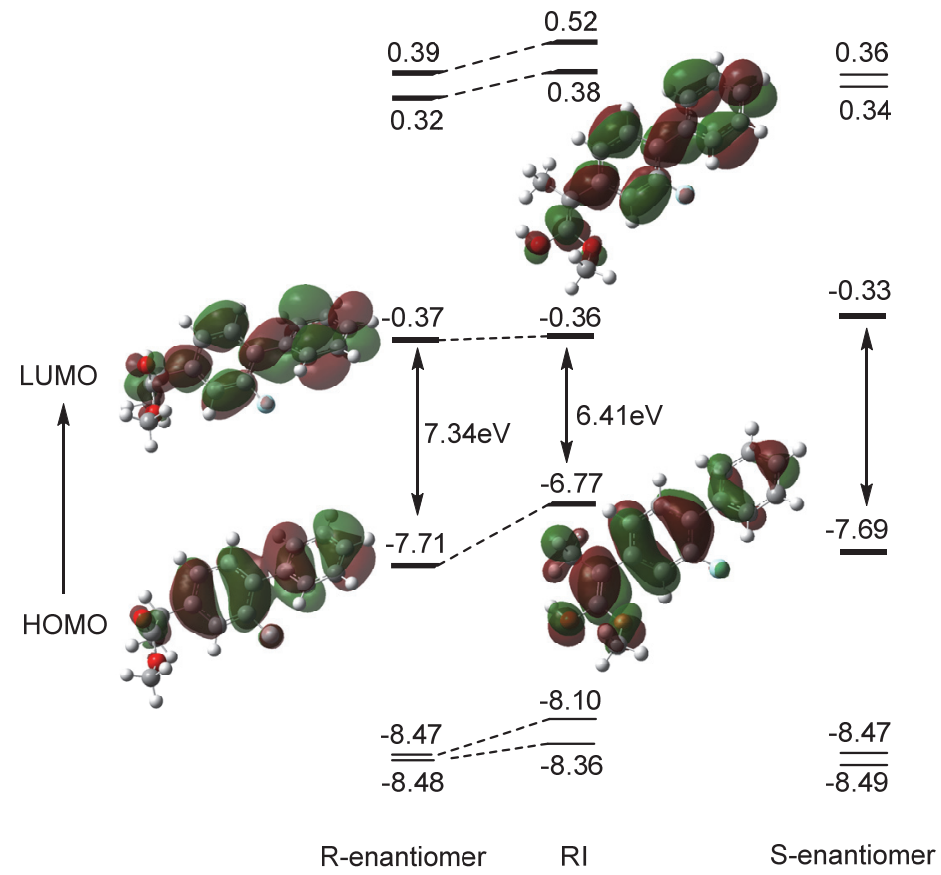

Fig. 5. TD-PBE0/6-31++G(d,p) computed orbitals of the $(R)$ and $(S)$ flurbiprofen methyl ester and (E)-2-(2-fluoro-[1,1'-biphenyl]-4-yl)-1-methoxyprop-1-en-1-ol

It can be concluded from the IRC calculations that this transition state leads to (R)-flurbiprofen methyl ester in one direction and to an $E$ isomer region corresponding to a 1-en-1-ol in the other. In 
addition, we used the similar calculation method to optimize the intermediate $(E)-2$-(2-fluoro-[1,1'biphenyl]-4-yl)-1-methoxyprop-1-en-1-ol, where the methyl group is twisted by about $90^{\circ}$ counterclockwise, relative to $(R)$-flurbiprofen methyl ester. Fig. 6. displays the optimized structures of both reactive intermediate $(R I)$ and $(R)$-flurbiprofen methyl ester.

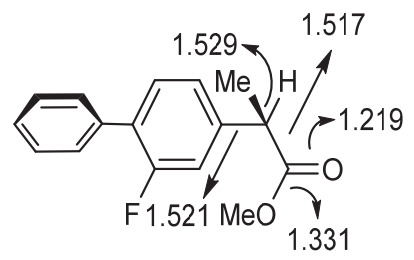

(R)-Flurbiprofen methyl ester

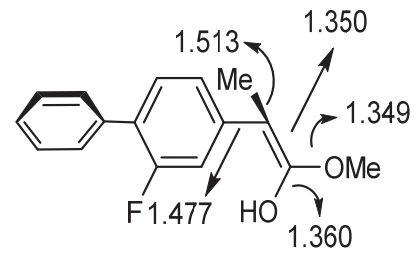

(E)-2-(2-fluoro-[1,1'-biphenyl]-4-yl)-1-methoxyprop-1-en-1-ol

Fig. 6. The optimized structures and bond length (in $\AA$ ) of $(R)$-flurbiprofen methyl ester and intermediate (E)-2-(2-fluoro-[1,1'-biphenyl]-4-yl)-1-methoxyprop-1-en-1-ol

From the comparison of RI with TS1 and $(R)$-flurbiprofen methyl ester, it can be seen that there are some bond length changes in geometries. The obtained results clearly demonstrate the elongation of the $\mathrm{C} 15-\mathrm{O} 18$ bond length (corresponding to hydrogen transfer) from $1.219 \AA$ in the $\mathrm{R}$ ester to 1.287 and $1.360 \AA$ in the TS1 and RI respectively. This elongation is the most important structural change for the TS1 and RI. Aso, the H17-O18 distance changes from $2.538 \AA$ in initial R isomer to $1.221 \AA$ in the TS1 and finally to $0.968 \AA$ in RI with a calculated bond advancement (1) of $0.78 .{ }^{27,28}$ In addition, the C14-C3 bond length reduced from $1.525 \AA$ in the $\mathrm{R}$ ester to 1.472 and $1.477 \AA$ in the TS1 and RI respectively. Further, $\mathrm{C} 14-\mathrm{C} 15$ bond length is also reduced from $1.517 \AA$ in the R ester to 1.450 and $1.349 \AA$ in the TS1 and RI respectively. Additionally, the C15-O19 bond length increased from 1.331 in the R ester to $1.349 \AA$ in RI. Increasing the C15-O19 bond length and decreasing the value of C14$\mathrm{C} 15$ are attributed to the change of the resonance cycle with $\mathrm{O} 18-\mathrm{C} 15-\mathrm{O} 19$ to $\mathrm{Ar}^{1}-\mathrm{C} 14-\mathrm{C} 15$. Furthermore, this issue has been confirmed by the difference between the HOMO and LUMO of $(R)$ flurbiprofen methyl ester and RI. ${ }^{29}$ The calculations revealed that due to the extending of conjugated system in $R I$ species, the HOMO-LUMO energy gap value reduced from $7.34 \mathrm{eV}$ to $6.41 \mathrm{eV}$. The $R I$ possesses $10.84 \mathrm{kcal} / \mathrm{mol}$ higher energy than $(R)$-flurbiprofen methyl ester hence, the species has a tendency to second $\mathrm{H} 17$ migration. The result of second migration of $\mathrm{H} 17$ from $\mathrm{O} 18$ to $\mathrm{C} 14$ along with $90^{\circ}$ anticlockwise rotating of methyl group after passing through the transition state TS2 is generation of stable $(S)$-flurbiprofen methyl ester.

As aforementioned, the symmetry of [1,3]-hydrogen shift from $\mathrm{C} 15$ to $\mathrm{C} 14$ is forbidden. ${ }^{23-25}$ Due to this symmetry forbidden, the migration of $\mathrm{H} 17$ goes to completion by the excitation of $R I$ to the first excited singlet state $\mathrm{S} 1$. From the calculations, it can be understood that for $R I$ species the excitation to the $\mathrm{S} 1$ state occurs at $285.25 \mathrm{~nm}$ with oscillator strength $\mathrm{f}=0.8825$. Moreover, several excitations were observed at position $256.85,249.14,238.36,227.45$ and $225.25 \mathrm{~nm}$ with oscillator strengths $\mathrm{f}=0.0155$, $\mathrm{f}=0.0358, \mathrm{f}=0.0010, \mathrm{f}=0.0025$ and $\mathrm{f}=0.1102$, respectively. The results depicted that the $R I$ singlet excited system after passing through the TS2 results in formation of $(S)$ - flurbiprofen methyl ester. The calculations revealed that the TS2, that corresponds to the migration of $\mathrm{H} 17$ from $\mathrm{C} 15$ to $\mathrm{C} 14$ lies 71.64 $\mathrm{kcal} / \mathrm{mol}$ higher in energy than optimized ground state $(R)$-flurbiprofen methyl ester. The Stereochemistry of this reaction is dominated by internal methylene rotations which favor inversion of stereochemistry at C14. The comparison of TS2 with RI and $(S)$-flurbiprofen methyl ester shows a significant reduction of the $\mathrm{C} 15-\mathrm{O} 18$ bond length from $1.360 \AA$ in the RI to $1.286 \AA$ in TS2 and finally to $1.217 \AA$ at the product $(S)$-flurbiprofen methyl ester. In addition, both the $\mathrm{C} 14-\mathrm{C} 15$ and $\mathrm{C} 14-\mathrm{C} 3$ bond lengths show gradual elongation of $1.350 \AA$ in RI to 1.448 and $1.517 \AA$ in TS2 and $(S)$-flurbiprofen methyl ester for C14-C15, and for C14-C3 bond length from $1.477 \AA$ in RI to 1.480 and $1.519 \AA$ in

\footnotetext{
${ }^{1}$ Aromatic
} 
TS2 and (S)-flurbiprofen. Also, the H17-C14 bond changes from $1.575 \AA$ in the TS2 to $1.090 \AA$ in $(S)$ flurbiprofen methyl ester. The calculated 1 of 0.55 for finally H17-C14 bond formation demonstrates that the H17-O18 with $1=0.78$ in step one forms much more rapidly than the H17-C14 bond formation. As illustrated in Fig. 3 we observed a second transition state TS1' for cleaving the O18-H17. Our computations showed that this transition state lies $70.94 \mathrm{kcal} / \mathrm{mol}$ higher in energy than $(R)$-flurbiprofen methyl ester and will convert the $R I$ to the primary $R$ isomer. As can be seen in Fig. 7 this transition state (TS1') and the transition state TS1 have similar geometry with the same free energy.
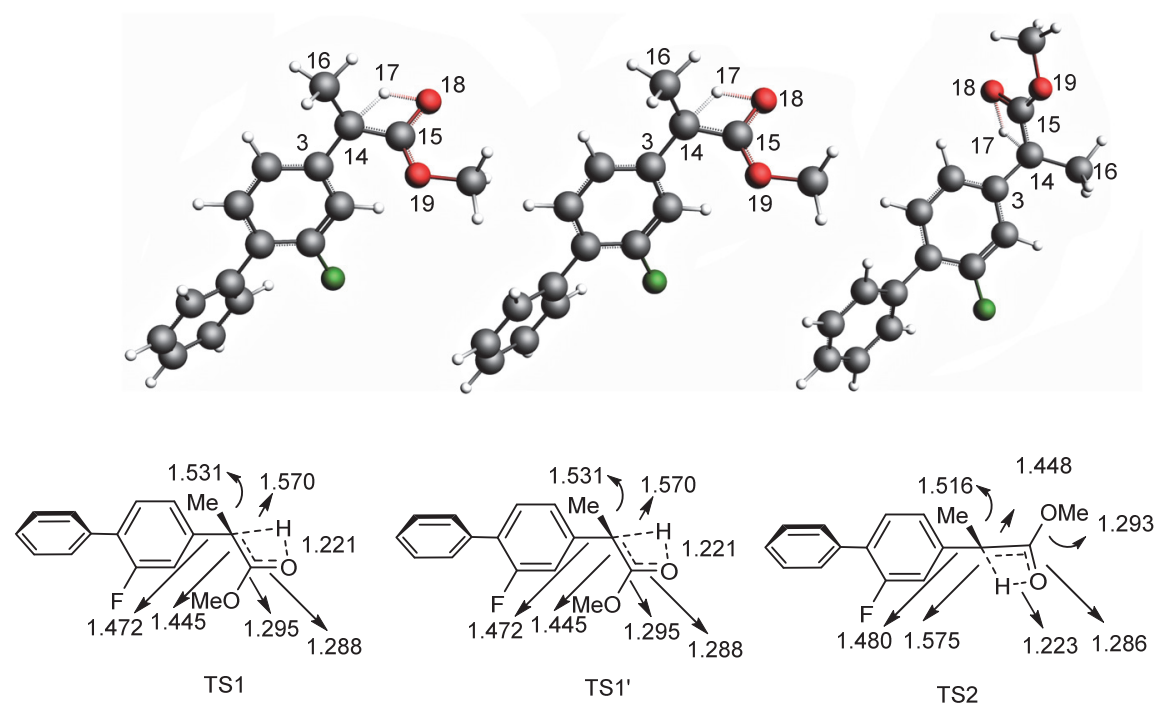

Fig. 7. The optimized structures and bond length (in $\AA$ ) of TS1, 1' and 2

The IRC calculations on TS2 and TS1' clearly shows that both the transition states 2 and 1' lead to $R I$ species in one direction but, in the other direction the transition state 2 leads to $(S)$-flurbiprofen methyl ester while the transition state 1 ' ends up to the initial $(R)$-flurbiprofen methyl ester. We found out that the difference in the barrier for inversion and retention is insignificant. But, the $-1.50 \mathrm{kcal} / \mathrm{mol}$ of thermodynamic stability of the $(S)$-flurbiprofen methyl ester is in agreement with the experimental preference for inversion. This result is also validated by $-1.63 \mathrm{kcal} / \mathrm{mol}$ of $(S)$-flurbiprofen methyl ester stability than the $R$ )-flurbiprofen methyl ester in CBS-4M level of theory.

\subsection{Mechanistic cycle for isomerization of racemic flurbiprofen}

As can be seen, the mechanism of isomerization and the results of our studies are shown in Scheme 1. We proposed three steps for isomerization reaction including (1), racemic flurbiprofen Fischer esterification to racemic flurbiprofen methyl ester, (2), $(R)$-flurbiprofen methyl ester isomerization to $(S)$-flurbiprofen methyl ester and (3), kinetic enzymatic resolution employing Candida rugosa lipase to convert the generated $(S)$-flurbiprofen methyl ester to $(S)$-flurbiprofen. The formed $(S)$-flurbiprofen is extracted by using Candida rugosa lipase to out of cycle. Then the remaining $(R)$-flurbiprofen methyl ester is injected continuously into the isomerization cycle. From the obtained results, it can be found out that the energies of transition state 1,1 ' and 2 are the same and all three are less energy barrier than first excited singlet state $(R)$-flurbiprofen methyl ester. The obvious conclusion to be drawn from the above findings is that the conversion cycle is controlled and restricted by initial excitation energy. 


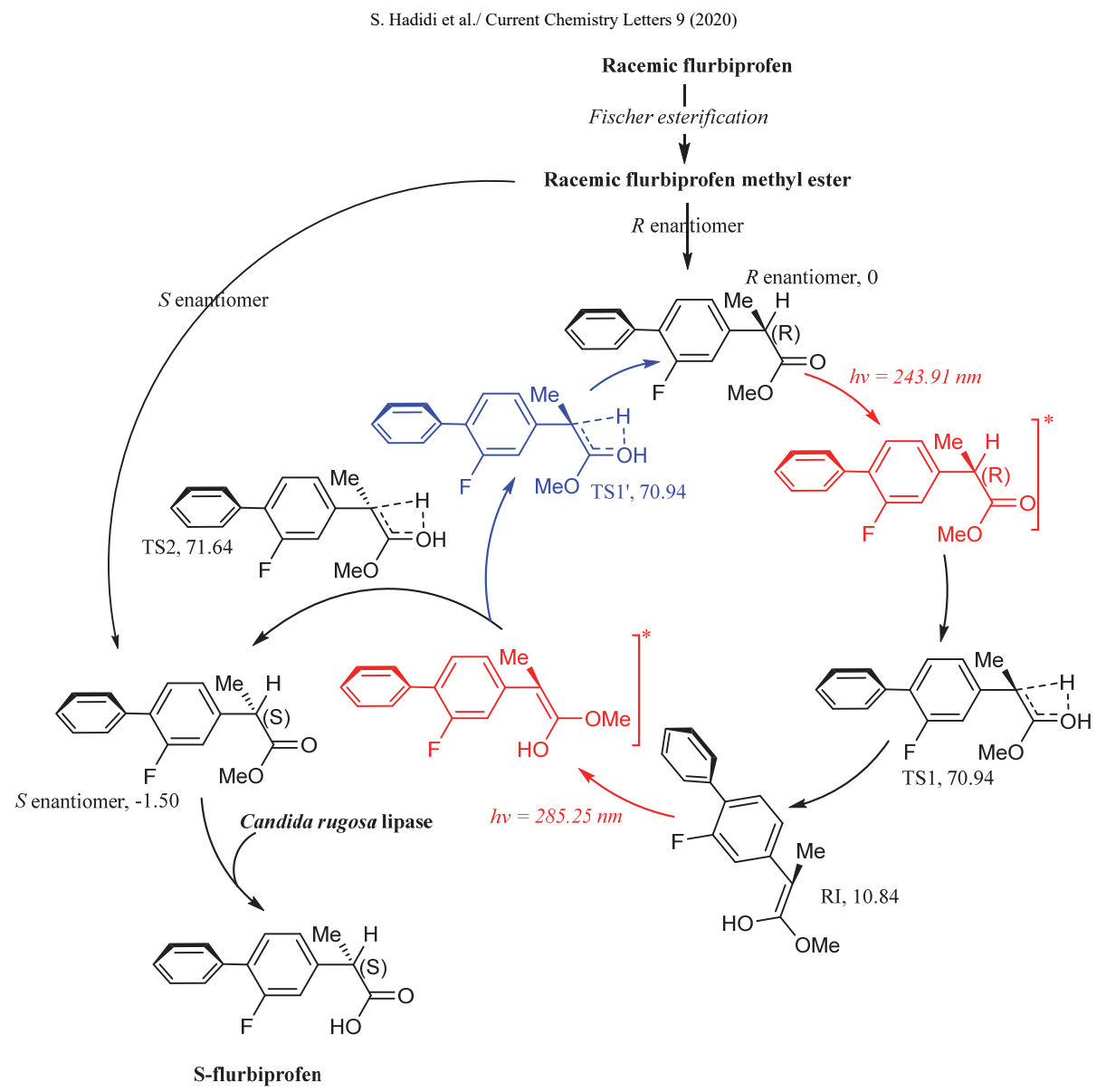

Scheme 1. The isomerization mechanistic cycle of racemic flurbiprofen

\section{Conclusions}

The isomerization of flurbiprofen was studied theoretically by employing the DFT method. The obtained results suggested that the conversion cycle is probably limited and controlled by initial excitation energy of $(R)$-flurbiprofen methyl ester. Also, it can be understood from the scanning of C14H17 distance on the excited singlet surfaces that the reaction proceeds with a relatively stable intermediate. The structural analysis of the intermediate system clearly revealed the significant impact of Ar-C14-C15 resonance on intermediate stability. The intermediate can be converted to $(R)$ or $(S)$ enantiomers with the barrier of approximately $71 \mathrm{kcal} / \mathrm{mol}$ related to $(\mathrm{R})$-flurbiprofen methyl ester. The calculations depicted that the $(S)$-enantiomer is more stable than the $(R)$ isomer. With respect to this issue, it is predicted that the intermediate will be preferentially converted to $(S)$-enantiomer with the $1.50 \mathrm{kcal} / \mathrm{mol}$ more stability. These data can justify the more photostable of $(S)$-flurbiprofen methyl ester than $(R)$ - enantiomer.

\section{Computational details}

In current investigation, the ORCA ${ }^{30}$ was utilized in order to perform the DFT calculations. ${ }^{31}$ The Berny algorithm was employed to examine the transition structures with an imaginary frequency. We authenticated all transition structures via scanning the potential energy surface and intrinsic reaction coordinate (IRC) calculations. For valence excitations of present system, exchange-correlation functionals with very high Hartree-Fock composition always tend to evidently overestimate excitation energy. ${ }^{32}$ PBE0 should be one of the best choices for obtaining relatively accurate excitation energy for present system. So the calculations were carried out by employing the pure functional of Perdew, Burke and Ernzerhof ${ }^{33,34}$ with $6-31+\mathrm{G}(\mathrm{d}, \mathrm{p})$ basis set without any symmetry restriction. In order to extract thermal corrections to the Gibbs free energies, frequency analyses were performed at the same level of 
theory. All the calculations were carried out for water based on the SMD variation of IEFPCM of Truhlar and workers ${ }^{35}$ via the SMD option. Further, the degrees of bonds advancement were estimated based on the following equation: ${ }^{36,37}$

$$
l_{X-Y}=1-\left(r_{X-Y}^{T S}-r_{X-Y}^{P}\right) / r_{X-Y}^{P}
$$

where $r_{X-Y}^{T S}$ is the distance between the reaction centers $X$ and $Y$ at the transition structure and $r_{X-Y}^{P}$ is the same distance at the corresponding product. The atom numbering utilized throughout the study is illustrated in Fig. 1.

\section{References}

1 Bhasin R., Bhuttani P., Krishnan C., and Sachdev S. (1979) Flurbiprofen and phenylbutazone in rheumatoid arthritis and osteoarthrosis: A double-blind study. The Journal of the Association of Physicians of India, 27 (5), 379-383.

2 Kantor T. G. (1986) Physiology and treatment of pain and inflammation: Analgesic effects of flurbiprofen. The American journal of medicine, 80 (3), 3-9.

3 Frölich J. (1997) A classification of nsaids according to the relative inhibition of cyclooxygenase isoenzymes. Trends in Pharmacological Sciences, 18 (1), 30-34.

4 Flower R. J. (2003) The development of cox2 inhibitors. Nature Reviews Drug Discovery, 2 (3), 179.

5 Greig M. E., and Griffin R. L. (1975) Antagonism of slow reacting substance in anaphylaxis (srsa) and other spasmogens on the guinea pig tracheal chain by hydratropic acids and their effects on anaphylaxis. Journal of medicinal chemistry, 18 (1), 112-116.

6 Shimura K., Oto A., Hanai Y., Watanabe S., Toda M., Asada K., Ishibashi K., Shimabukuro Y., Yokochi N., and Shiramizu H. (1981) Analgesic effect of fentiazac after tooth extraction or minor oral surgery. Clinical therapeutics, 4 (1), 12-17.

7 Muscat J. E., Stellman S. D., and Wynder E. L. (1994) Nonsteroidal antiinflammatory drugs and colorectal cancer. Cancer, 74 (7), 1847-1854.

8 Hadidi S., Shiri F., and Norouzibazaz M. (2019) A dft study of the degradation mechanism of anticancer drug carmustine in an aqueous medium. Structural Chemistry, 30 (4), 1315-1321.

9 Hadidi S., Shiri F., and Norouzibazaz M. (2020) Mechanistic study of fenoprofen photoisomerization to pure (s)-fenoprofen: A dft study. Structural Chemistry, 31 (1), 115-122.

10 Hadidi S., Shiri F., and Norouzibazaz M. (2019) Conversion mechanism and isomeric preferences of the cis and trans isomers of anti-cancer medicine carmustine; a double hybrid dft calculation. Chemical Physics, 522, 39-43.

11 Hadidi S., Shiri F., and Norouzibazaz M. (2020) Theoretical mechanistic insight into the gabapentin lactamization by an intramolecular attack: Degradation model and stabilization factors. Journal of pharmaceutical and biomedical analysis, 178, 112900.

12 Jamali F., Mehvar R., and Pasutto F. (1989) Enantioselective aspects of drug action and disposition: Therapeutic pitfalls. Journal of pharmaceutical sciences, 78 (9), 695-715.

13 Evans A. M. (1992) Enantioselective pharmacodynamics and pharmacokinetics of chiral nonsteroidal anti-inflammatory drugs. European journal of clinical pharmacology, 42 (3), 237-256.

14 Gaut Z. N., Baruth H., Randall L., Ashley C., and Paulsrud J. (1975) Stereoisomeric relationships among anti-inflammatory activity, inhibition of platelet aggregation, and inhibition of prostaglandin synthetase. Prostaglandins, 10 (4), 59-66.

15 Geisslinger G., Muth-Selbach U., Coste O., Vetter G., Schrödter A., Schaible H. G., Brune K., and Tegeder I. (2000) Inhibition of noxious stimulus-induced spinal prostaglandin e 2 release by flurbiprofen enantiomers: A microdialysis study. Journal of neurochemistry, 74 (5), 2094-2100.

16 Andersen J. V., and Hansen S. H. (1992) Simultaneous determination of (r)-and (s)-naproxen and (r)-and (s)-6-o-desmethylnaproxen by high-performance liquid chromatography on a chiral-agp column. Journal of Chromatography B: Biomedical Sciences and Applications, 577 (2), 362365 . 
17 Jamali F., Berry B. W., Tehrani M. R., and Russell A. S. (1988) Stereoselective pharmacokinetics of flurbiprofen in humans and rats. Journal of pharmaceutical sciences, 77 (8), 666-669.

18 Chen C. S., and Sih C. J. (1989) General aspects and optimization of enantioselective biocatalysis in organic solvents: The use of lipases [new synthetic methods (76)]. Angewandte Chemie International Edition in English, 28 (6), 695-707.

19 Mustranta A. (1992) Use of lipases in the resolution of racemic ibuprofen. Applied microbiology and biotechnology, 38 (1), 61-66.

20 Bornscheuer U. T., and Kazlauskas R. J. (2006) Hydrolases in organic synthesis: Regio-and stereoselective biotransformations, Ed, John Wiley \& Sons.

21 Ghanem A., and Aboul-Enein H. Y. (2005) Application of lipases in kinetic resolution of racemates. Chirality: The Pharmacological, Biological, and Chemical Consequences of Molecular Asymmetry, 17 (1), 1-15.

22 Chen C.-S., Shieh W.-R., Lu P.-H., Harriman S., and Chen C.-Y. (1991) Metabolic stereoisomeric inversion of ibuprofen in mammals. Biochimica et Biophysica Acta (BBA)-Protein Structure and Molecular Enzymology, 1078 (3), 411-417.

23 Gill G. (1968) The application of the woodward-hoffmann orbital symmetry rules to concerted organic reactions. Quarterly Reviews, Chemical Society, 22 (3), 338-389.

24 Sato D., Shiba T., Karaki T., Yamagata W., Nozaki T., Nakazawa T., and Harada S. (2017) X-ray snapshots of a pyridoxal enzyme: A catalytic mechanism involving concerted [1,5]-hydrogen sigmatropy in methionine $\gamma$-lyase. Scientific Reports, 7 (1), 4874.

25 ŠOlomek T. S., Ravat P., Mou Z., Kertesz M., and JuríČEk M. (2018) Cethrene: The chameleon of woodward-hoffmann rules. The Journal of organic chemistry, 83 (8), 4769-4774.

26 Woodward R. B., and Hoffmann R. (1965) Selection rules for sigmatropic reactions. Journal of the American Chemical Society, 87 (11), 2511-2513.

27 Demchuk O. M., Jasiński R., and Pietrusiewicz K. M. (2015) New insights into the mechanism of reduction of tertiary phosphine oxides by means of phenylsilane. Heteroatom Chemistry, 26 (6), 441-448.

28 Jasiński R. (2015) A stepwise, zwitterionic mechanism for the 1, 3-dipolar cycloaddition between (z)-c-4-methoxyphenyl-n-phenylnitrone and gem-chloronitroethene catalysed by 1-butyl-3methylimidazolium ionic liquid cations. Tetrahedron Letters, 56 (3), 532-535.

29 Cortizo-Lacalle D., Howells C. T., Pandey U. K., Cameron J., Findlay N. J., Inigo A. R., Tuttle T., Skabara P. J., and Samuel I. D. (2014) Solution processable diketopyrrolopyrrole (dpp) cored small molecules with bodipy end groups as novel donors for organic solar cells. Beilstein journal of organic chemistry, 10 (1), 2683-2695.

30 Neese F., and Wennmohs F. (2013) Orca (3.0. 2)-an ab initio. DFT and semiempirical SCF-MO package,(Max-Planck-Institute for Chemical Energy Conversion Stiftstr. 34-36, 45470 Mulheim ad Ruhr, Germany).

31 Becke A. D. (1993) Density-functional thermochemistry. Iii. The role of exact exchange. The Journal of chemical physics, 98 (7), 5648-5652.

32 Jacquemin D., Mennucci B., and Adamo C. (2011) Excited-state calculations with td-dft: From benchmarks to simulations in complex environments. Physical chemistry chemical physics, 13 (38), 16987-16998.

33 Perdew J. P., Burke K., and Ernzerhof M. (1996) Generalized gradient approximation made simple. Physical review letters, 77 (18), 3865.

34 Perdew J., Burke K., and Ernzerhof M. (1996) Phys rev lett 77: 3865. Errata:(1997) Phys Rev Lett, 78, 1396.

35 Marenich A. V., Cramer C. J., and Truhlar D. G. (2009) Universal solvation model based on solute electron density and on a continuum model of the solvent defined by the bulk dielectric constant and atomic surface tensions. The Journal of Physical Chemistry B, 113 (18), 6378-6396.

36 Jasiński R. J. T. L. (2015) A stepwise, zwitterionic mechanism for the 1, 3-dipolar cycloaddition between (z)-c-4-methoxyphenyl-n-phenylnitrone and gem-chloronitroethene catalysed by 1butyl-3-methylimidazolium ionic liquid cations. 56 (3), 532-535. 
37 Demchuk O. M., Jasiński R., and Pietrusiewicz K. M. J. H. C. (2015) New insights into the mechanism of reduction of tertiary phosphine oxides by means of phenylsilane. 26 (6), 441448.

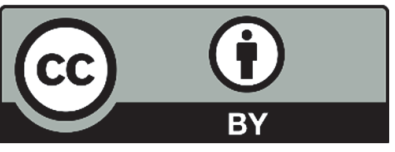

(C) 2020 by the authors; licensee Growing Science, Canada. This is an open access article distributed under the terms and conditions of the Creative Commons Attribution (CC-BY) license (http://creativecommons.org/licenses/by/4.0/). 